\title{
Impact of Rosemary Leave extracts on Triacylglycerol levels in Human Serum Albumin
}

\author{
Zeyad J Yassin* and Tarek M Zaida* \\ Department of Chemistry, Gaza \\ *Corresponding author: Zeyad J Yassin, Department of Chemistry, Gaza \\ Tarek M Zaida, Department of Chemistry, Gaza
}

\section{ARTICLE INFO}

Received: 幽 November 25, 2019

Published: December 04, 2019

Citation: Zeyad J Yassin, Tarek M Zaida. Impact of Rosemary Leave extracts on Triacylglycerol levels in Human Serum Albumin. Biomed J Sci \& Tech Res 23(4)2019. BJSTR. MS.ID.003921.

Keywords: Rosemary Plant; Human Serum Albumin; Triacylglycerol; Relative Affinity

Abbreviations: TAG: Triacylglycerol; RM: Rosemary; HAS: Human Serum Albumin

\section{ABSTRACT}

In order to estimate the best affinity of rosemary extract towards the removal of triacylglycerol (TAG) from a real human serum albumin sample HSA in vitro, Rosemary leaves were obtained from Rosmarinus officinalis $L$ plant, and rosemary extracts were prepared with four well selected ethanol-water concentrations combinations and were screened accordingly. The relative affinities of the rosemary (RM) extracts using the selected extraction solvents were evaluated spectrophotometrically and analyzed by applying a well-defined standard equation for each extract. Our results of the current study suggest that the extracts of RM plant could be considered as effective agents to remove TAG from human serum albumin in vitro. The relative affinity is dependent on the concentration of ethanol - water ratio used in the extraction procedure. The relative affinities of plant extracts in this study towards the removal of TAG showed different extends for TAG removal and were varied from 3.96 to 11.16 dependent on ethanol - water ratio. The studied extracts interacted with human serum albumin (HSA) with different binding affinities, which were directly related to their relative affinities to remove TAG from the human serum albumin sample. The present study clearly shows that the highest relative affinity was observed in $50 \%$ ethanol - water extracting solution.

\section{Introduction}

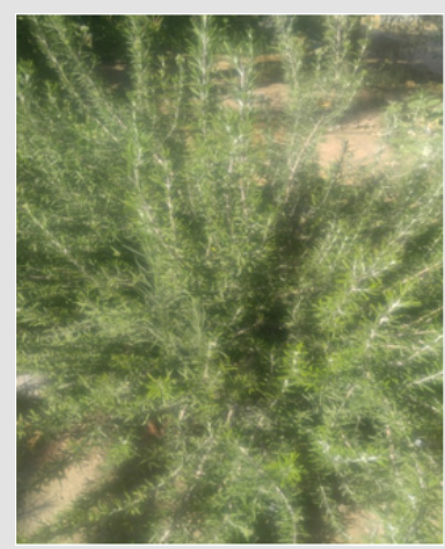

Figure 1: Illustration of Rosemary plant (Rosmarinus officinalis L.).

The management of many chronic diseases such as hypertension, Diabetes Mellitus, obesity, has been done by some known medicinal plants for a long time by different nations [1]. In Asia, Africa, Europe and the Americas. Medicinal herbs as medicines have played an important role in treating different diseases [2]. The use of medicinal plants in the traditional medicine is on the rise because of their treatment properties of various diseases [3]. The advantage of herbal medicine rises from the fact that plants contain only natural substances in contrast to synthetic drugs, which have been proved to have major side effects despite their treatment ability. Several herbs have been proved efficient in reducing high blood cholesterol, blood sugar, providing some protection against cancer and stimulating the immune system [4]. Figure 1 shows the commonly called Rosemary plant (Rosmarinus officinalis L.) native to the north and south coasts of the Mediterranean Sea, is an evergreen perennial aromatic shrub belonging to the family Labiatae [5]. Some of common uses of Rosemary is as a spice and flavoring agent in food processing [6]. It has been found that dried 
leaves and flowers of Rosemary are considered to be an interesting source of biologically active phytochemicals such as phenolic com- pounds including carnosol, carnosic acid, rosmarinic acid (Figure 2 ) and others, having in vitro and in vivo antioxidant activity $[7,8]$.

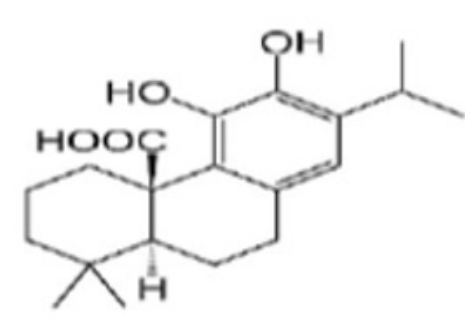

Carnosic Acid

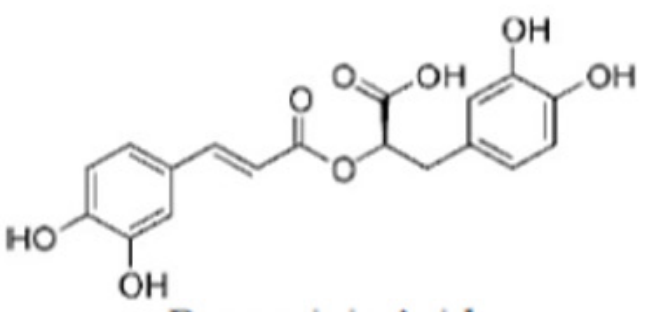

Rosmarinic Acid

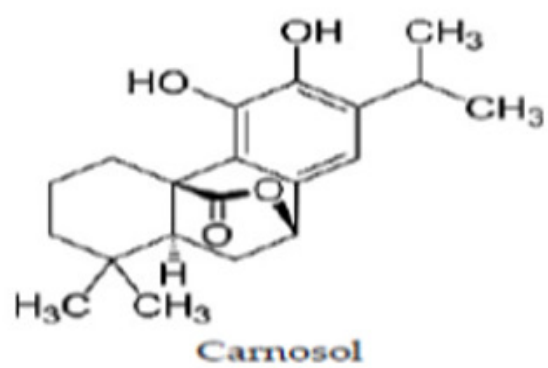

Figure 2: The structure of major polyphenols found in of Rosmarinus officinalis.

Some antioxidant phenolics contained in Rosemary have been shown to provide a defensive ability against oxidative stress generated from different metabolic activities of the cells [9]. Rosemary also demonstrates antioxidant, diuretic, antiinflammatory, antimicrobial, anti-carcinogenic, hypoglycemic and hypolipidimic activities according to many studies $[10,11]$. The present study was designed to evaluate the effects of rosemary extract on blood triacylglycerol (TAG) level bound to human serum albumin as there is a strong evidence for extract from Rosemary leaves to play a role as a hypoglycemic and a hypolipidemic agent. RM plant extracts prepared in selected ethanol-water concentrations of compositions $(0 \%, 30 \%, 50 \%$ and $100 \% \mathrm{v} / \mathrm{v}$ ) from some species of rosemary leaves were screened for TAG removal from human serum albumin sample. To maximize the recovery of RM extract for TAG removal from human serum albumin, establishing optimal and specific extraction condition using binary solvent system of ethanol and water is important. Our findings may provide some useful information to support the age-long practice of using RM as a potential anti-hypertensive medicinal herb [12].

\section{Materials and Methods}

Materials and Solutions HSA (fatty acid free, 96 99\%) is purchased from Sigma-Aldrich and prepared in 50mM potassium phosphate buffer solution ( $\mathrm{pH} 7.0$ ) containing $20 \mathrm{mM} \mathrm{NaCl}$ solution and stored in the dark at $4^{\circ} \mathrm{C}$. Potassium phosphate buffer salts were obtained from Merck. Other chemicals are of analytic grade. UV-Vis Absorbance Spectroscopy. Absorption spectra are obtained with a JASCO-V550 spectrophotometer. The scan rate was kept at $200 \mathrm{~nm} / \mathrm{min}$ for all analysis at 298K in phosphate buffer (pH 7.0) across $200-550 \mathrm{~nm}$ using a $1 \mathrm{~cm}$ quartz cell.

\section{Plant Leaves Collection and Preparation}

Rosemary leaves were bought from a local market in Gaza city, Palestine in September 2018. The leaves were washed with water to get rid of residual soil and dried. The collected RM leaves were shade, dried and then were incubated at $40^{\circ} \mathrm{C}$ for 4 hours until complete drying, and finally were ground to fine powder using a hammer mill. The fine powder of Rosemary Leaves was then stored in airtight plastic containers.

\section{Rosemary Extraction}

A quantity of 10 grams of fine ground powder of rosemary plant material were soaked in $100 \mathrm{ml}$ of boiled water, after $1 \mathrm{~h}$ stirring, at room temperature overnight, the supernatant was decanted. The pooled supernatant was combined and filtered and then was placed in a porous bag or "thimble" made of strong filter paper, which is placed in Soxhlet chamber. The extracting solvent (under study: $100 \%$ ethanol, $50 \%, 30 \%$ or $0 \%$ ) in Soxhlet flask was then heated, and its vapors condensed in Soxhlet condenser. The condensed extract dripped into the thimble containing the crude leaves extract, was then collected, filtered, and the solvent was evaporated to the minimum volume (few mls). The different ethanolic extracts of RM leave plant samples were stored at $4^{\circ} \mathrm{C}$ in the absence of light for further experiments. The advantage of this method, compared to other normal methods, is that large amounts of plant extract can be eluted with a much smaller quantity of solvent.

\section{Spectroscopic Analysis}

The absorbance of UV-vis spectra was recorded along the wavelength range of $240-400 \mathrm{~nm}$, in $10 \mathrm{~mm}$ quartz cuvettes by the spectrophotometer. The scan rate was kept at $200 \mathrm{~nm} / \mathrm{min}$ for all analysis. The triacylglycerol-containing HSA was analyzed with and without crude rosemary extract using a simple two-site model [6] in which the vital component in the crude plant extract was expected to interact with binding sites on a protein (HSA).

\section{Analysis Method}

UV-vis absorption spectra experiments were performed to identify the extent of TAG removal from HSA sample as a result of addition of the RM extracts. UV-vis absorption measurement is a very simple method to know the complex information formed and usually applied to explore the structural change. we used the difference absorption spectroscopy signals to analyze the spectra, the UV-vis absorption spectra of HSA and the difference in the 
absorption spectra of HSA/TAG in presence and absence of RM extracts at the same concentrations.

\section{Results and Discussion}

In this study, ethanol/water formulations of compositions ( $0 \%, 30,50 \%$ and $100 \%$ ) were chosen as extraction solvents because ethanol/water formulations are widely used to obtain crude extracts of phytochemicals from plant materials in the herbal medicine industry for therapeutic applications [13]. UV absorption measurement is a very simple method and applicable to explore the complex formation in the present work $[14,15]$. The UV spectra of HSA with the absence and presence of RM extracts in different ethanol-water solvents were performed (Figures 3a-3d). It revealed that the peak intensity of HSA rose with the addition of the selected RM extract. The results demonstrated that the absorption values of free HSA were obviously lower than those of HAS- extract mixed solutions with the increasing concentrations of RM extract. These results indicated that there was an interaction between RM extract and HSA. Figure 3a represents $100 \%$ aqueous solvent. Figure 3b represents $100 \%$ ethanolic. Figure $3 c$ represents $70 \%$ aqueous and 50\% ethanolic, while Figure 3d represents 50\% ethanolic. Two major absorption peaks at $280 \mathrm{~nm}$ (which corresponds to the absorbance due to aromatic amino acids (Trp, Tyr, and Phe in HSA) and the other around $450 \mathrm{~nm}$ which corresponds to the absorbance due to TAG in human serum albumin studied in addition to some feeble peaks in the region of 210-260 nm which are observed in the (Figures 3a-3d), and reflected the absorption of the backbone in the protein studied [16]. Both peak intensities were risen upon the addition of the RM extract aliquots. The values of the relative affinities $\left(\mathrm{K}_{\mathrm{r}}\right)$ for RM plant extracts towards removal of serum TAG from HSA sample were calculated according to the method described by published methods $[17,18]$ using equation (1) after recording the absorption readings at $455 \mathrm{~nm}$ which corresponds to TAG in HSA sample.

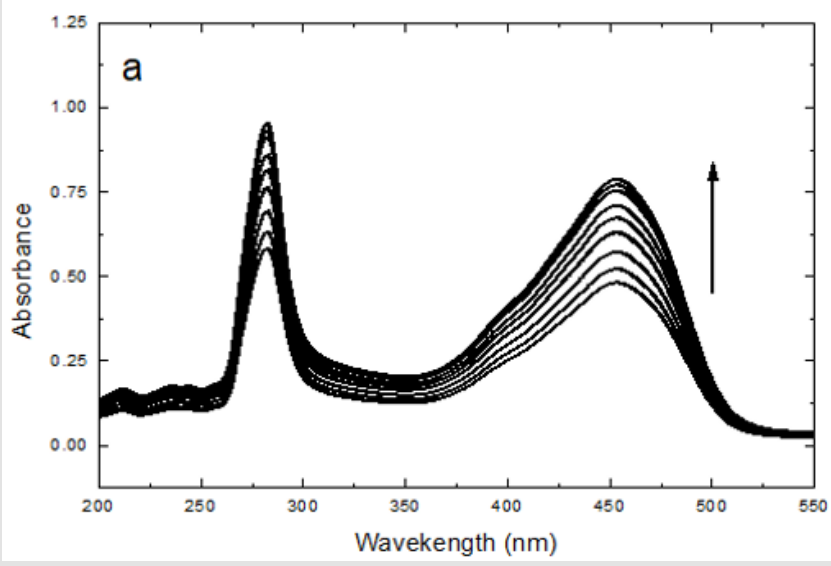

Figure 3a: UV absorption spectra of the RM extract in 100\% aqueous extracting solution. Arrow points to increasing concentrations of RM extract. The concentration of HSA was $15 \mu \mathrm{M}$. The arrow indicates successive addition of the $\mathrm{RM}$ extract. All in in phosphate buffer solution $(\mathrm{pH}=7.0)$ ,$T=298 \mathrm{~K}$.

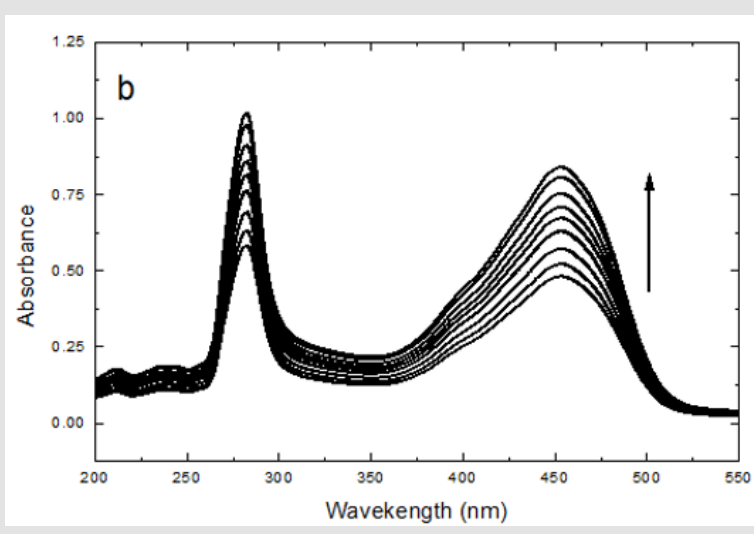

Figure 3b: The UV absorption spectra of the RM extract in $100 \%$ ethanol extracting solution. Arrow points to increasing concentrations of RM extract. The concentration of HSA was $15 \mu \mathrm{M}$. The arrow indicates successive addition of the RM extract. All in in phosphate buffer solution $(\mathrm{pH}$ $=7.0), T=298 \mathrm{~K}$

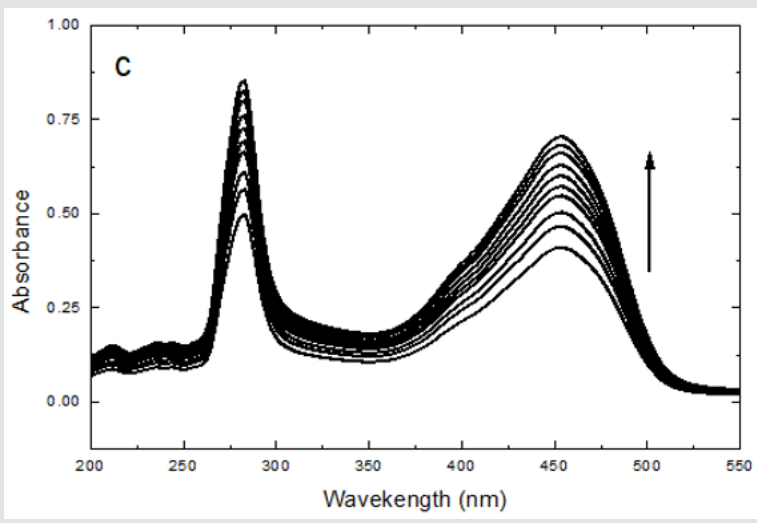

Figure 3c: The UV absorption spectra of the RM extract in $30 \%$ ethanol extracting solution. Arrow points to increasing concentrations of RM extract. The concentration of HSA was $15 \mu \mathrm{M}$. The arrow indicates successive addition of the $\mathrm{RM}$ extract. All in in phosphate buffer solution $(\mathrm{pH}=7.0)$ ,$T=298 \mathrm{~K}$.

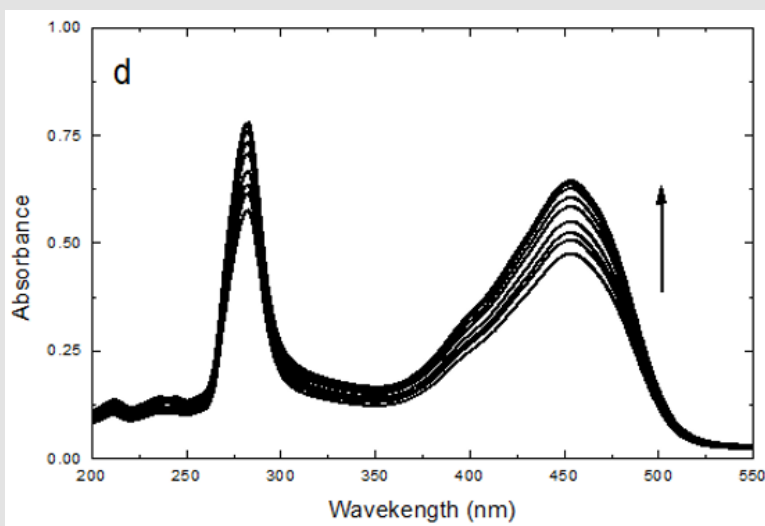

Figure 3d: The UV absorption spectra of the RM extract in $50 \%$ ethanol extracting solution. Arrow points to increasing concentrations of RM extract. The concentration of HSA was 15. The arrow indicates successive addition of the RM extract. All in in phosphate buffer solution $(\mathrm{pH}=7.0), T$ $=298 \mathrm{~K}$. 


$$
\frac{1}{\left(A-A_{0}\right)}=\frac{1}{\left(A_{\infty}-A_{0}\right)}+\frac{1}{K_{r}\left(A_{\infty}-A_{0}\right)} * \frac{1}{(\text { RMextrac })_{r, s}}
$$

Whereas,

A0 is the absorbance of HSA containing TAG at $455 \mathrm{~nm}$ in the absence of RM extract,

A $\infty$ is the final absorbance of the HSA - RM extract complex

A is the corresponding absorbance of complexes after each addition of RM extract aliquot.

$\mathrm{Kr}$ is the relative affinity constant for serum TAG removal.

And is the relative RM extract concentration dissolved in the proper extraction solvent.

Thus, the double reciprocal plot of $1 /(A-A 0) \mathrm{vs}$. $1 /(R M \text { extra })_{r, s}$ is linear and the relative affinity constant $\left(\mathrm{K}_{\mathrm{r}}\right)$ can be estimated from the ratio of the intercept to the slope $[17,18]$. The relative affinities $\left(\mathrm{K}_{\mathrm{r}}\right)$ of rosemary extract towards TAG removal from HSA were estimated by using UV-visible spectroscopy and analyzed using the mathematical model represented by equation (1) and the results were presented in Table 1 . As seen in Table 1 the ethanolic percent (in extracting solution) exhibited a contradictory effect, it shows an increasing trend in relative affinities $\left(\mathrm{K}_{\mathrm{r}}\right)$ of RM extract under study up to $50 \%$ and then descend. Rosemary extracts in different solvents exhibited different affinities towards the removal of TAG from HSA sample. The relative affinities of RM extracts (using ethanolic extraction solvents) towards removal of TAG follows the order: $100 \%$ water $\approx 100 \%$ ethanol $<30 \%$ ethanol $<50 \%$ ethanol. The following relative affinities 3.96, 4.05, 5.17 and 11.16 were recorded for RM extracts using: $100 \%$ aqueous, $30 \%$ aqueous, $100 \%$ ethanolic and $50 \%$ aqueous solutions, respectively. There was a remarkable difference for RM extract treated with $50 \%$ ethanolic/aqueous solution as shown in Table 1 . The data in Table 1 showed that RM extracted using a combination of ethanol/ water solvents revealed significantly higher affinity to remove serum TAG, this may be attributed due to the use of a polar solvent that may facilitate the interaction with the serum triglyceride. Interestingly ethanol alone exhibited a little removal ability of serum TAG. Among the extract solvents the synergistic solution in removing TAG, was the $50 \%$ ethanolic solution which demonstrated the highest affinity towards TAG removal from human serum albumin Figure 4.

Table 1: The relative affinities (Kr) for RM extract - HSA complexes.

\begin{tabular}{|c|c|}
\hline Relative affinity $\left(\mathbf{K}_{\mathbf{r}}\right)$ & Extraction solvent \\
\hline & Water $(\mathbf{v} / \mathbf{v}):$ Ethanol(v/v) \\
\hline 3.96 & $100 \%$ aqueous : $0 \%$ ethanolic \\
\hline 4.05 & $0 \%$ aqueous : $100 \%$ ethanolic \\
\hline 5.17 & $70 \%$ aqueous : $30 \%$ ethanolic \\
\hline 11.16 & $50 \%$ aqueous : $50 \%$ ethanolic \\
\hline
\end{tabular}

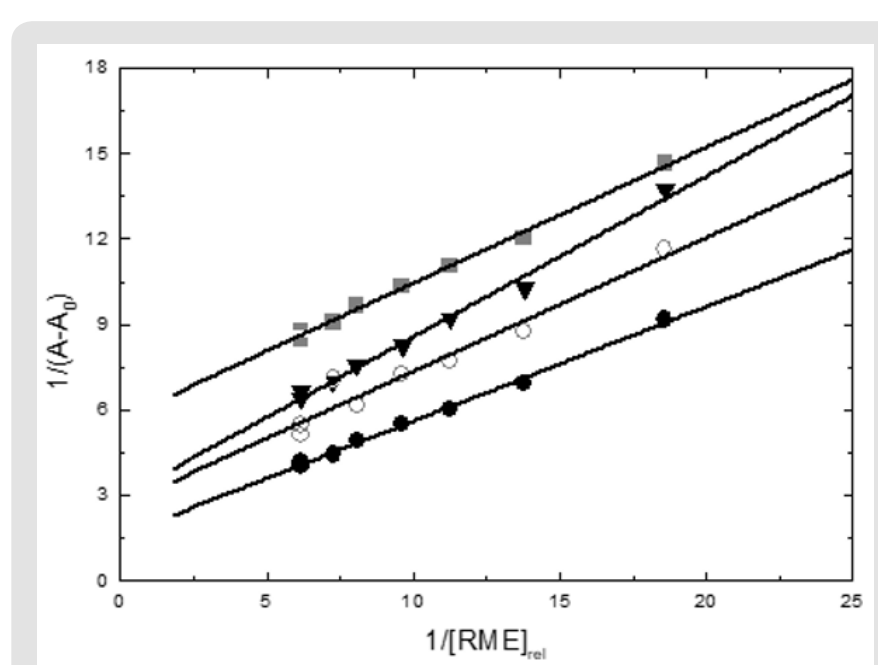

Figure 4: The double reciprocal Plots of $1 /(\mathrm{A}-\mathrm{A} 0)$ vs. $1 /[\mathrm{RM}] \mathrm{r}, \mathrm{s}$, for $\mathrm{RM}$ extracts and human serum TAG. (according to equation (1)).

\section{Conclusion}

In the present work, the interaction of RM extracts in different ethanol/water solvents (0,30,50 and 100\% ethanol) with HSA were investigated by UV-Vis absorption method. Experimental results suggest that RM extracts interact with HSA to varying extents. The relative affinities of RM extracts were determined. It was found that, the ratio of ethanol : water levels used in the extraction of RM leaves plays an important role to obtain optimum affinity towards removal of TAG from human serum albumin sample. The extent to which TAG was removed from HSA sample was estimated using a welldefined equation used for this purpose. Among the RM extracts in different extraction solvents, the extracting solution (50\% ethanol) exhibited the highest relative affinity towards removal of TAG from HSA sample. The results provide significant information for the role of RM extracts in removal of triacylglycerol in vivo and are expected to provide some useful information regarding researching medical application of herbal RM plant. Further clinical investigations are recommended to study the effect of RM extract on the removal of TAG from HSA in vivo in light of a recent study which found that the TAG levels were also decreased by $29.06 \%$ in the $10 \mathrm{~g} /$ day group and $21.3 \%$ and $14.97 \%$ in the $5 \mathrm{~g} /$ day and $2 \mathrm{~g} /$ day groups, respectively [19].

Also, the reduction in lipid levels observed after the consumption of RM has been suggested in other studies to be caused by a reduction in the absorption of dietary fat supported by an increase in fecal fat excretion [20]. Our findings support what has been found in a study that focused on the role of RM in decreasing total cholesterol level for all participants. The highest dose of 10 $\mathrm{g} /$ day tended to produce the highest reduction of TC by $34.48 \%$ followed by $5 \mathrm{~g}$ /day with reducsstion $17.97 \%$ and $2 \mathrm{~g} /$ day 11.48 . This reduction could be of clinical importance as its normalized TC values because TC value of more than $200 \mathrm{mg} / \mathrm{dL}$ could be linked to increased risk of heart and blood vessel diseases [21]. 


\section{References}

1. El Hilaly J, Adil T, Zafar HI, Badiâa LA (2007) Hypoglycemic, Hypocholesterolemic and Hypotriglyceridemic Effects of Continuous Intravenous Infusion of a Lyophilized Aqueous Extract of Ajuga iva l. Schreber Whole Plant in Streptozotocin-Induced Diabetic Rats. Pakistan Journal of Pharmaceutical Sciences 20: 261-268.

2. Wargovich MJ, Woods C, Hollis DM, Zander ME (2001) Herbals, Cancer Prevention and Health. Journal of Nutrition 131(11 Suppl): 3034S-3036S

3. Hussain Z, Waheed A, Qureshi R (2004) The Effect of Medical Plant's on Islamabad and Murree Rejoin of Pakistanon Insulin Secretion from INS1 Cell's. Journal of Phytotherapy Research 18(1): 73-77.

4. Abdul Rahim Al, Taha A (2011) Effects of Rosemary (Rosmarinus officinalis) on Lipid Profile of Diabetic Rats. Jordan Journal of Biological Sciences 4: 199-204.

5. Al Sereiti, MR, Abu Amer KM, Sen P (1999) Pharmacology of Rosemary (Rosmarinus officinalis Linn.) and Its Therapeutic Potentials. Indian Journal of Experimental Biology 37: 124-130.

6. Saito Y, Shiga A, Yoshida Y, Furuhashi T, Fujita Y (2004) Effects of Novel Gaseous Antioxidative System Containing a Rosemary Extract on the Oxidation Induced by Nitrogen Dioxide and Ultraviolet Radiation. Bioscience, Biotechnology, and Biochemistry 68(4): 781-786.

7. El Deeb KS (1993) Investigation of Tannin in Some Labiatae Species. Bulletin of Faculty of Pharmacy 31: 237-241.

8. Herrero M, Plaza M, Cifuentes A, Ibanez E (2010) Green Processes for the Extraction of Bioactives from Rosemary: Chemical and Functional Characterization via Ultra-Performance Liquid Chromatography-Tandem Mass Spectrometry and in-Vitro Assays. Journal of Chromatography 1217(16): 2512-2520

9. Matkowski A (2006) Plant Phenolic Metabolites as Antioxidants and Antimutagens. In Blume Y, Smertenko P, Durzan DJ (Eds.), UV Radiation, Nitric Oxide and Cell Death in Plants. NATO Life Science Monographs, IOS Press, Amsterdam, Netherlands 376: 129-148.

10. Dearlove RP, Greenspan P, Hartle DK, Swanson RB, Hargrove JL (2008) Inhibition of Protein Glycation by Extracts of Culinary Herbs and Spices. Journal of Medicinal Food 11(2): 275-281.

\section{ISSN: 2574-1241}

DOI: $10.26717 /$ BJSTR.2019.23.003921

Zeyad J Yassin, Tarek M Zaida. Biomed J Sci \& Tech Res

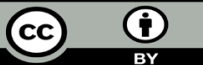

This work is licensed under Creative

Commons Attribution 4.0 License

Submission Link: https://biomedres.us/submit-manuscript.php
11. Nabekura T, Yamaki T, Hiroi T, Ueno K, Kitagawa S (2010) Inhibition of Anticancer Drug Efflux Transporter P-Glycoprotein by Rosemary Phytochemicals. Pharmacological Research 61(3): 259-263.

12. Madina Naimi , Filip Vlavcheski , Hesham Shamshoum, Evangelia Tsiani (2017) Rosemary Extract as a Potential Anti-Hyperglycemic Agent: Current Evidence and Future Perspectives. Nutrients 9(9): E968.

13. Wendakoon, Chitra, Peter Calderon, Daniel Gagnon (2012) Evaluation of Selected Medicinal Plants Extracted in Different Ethanol Concentrations for Antibacterial Activity against Human Pathogens. Journal of Medicinally Active Plants 1(2): 60-68.

14. Bi S, Song D, Tian Y, Zhou X, Liu Z (2005) Molecular spectroscopic study on the interaction of tetracyclines with serum albumins. Spectrochim Acta Part A 61(4): 629-636

15. Kandagal PB, Ashoka S, Seetharamappa J, Vani V, Shaikh SMT (2006) Study of the interaction between doxepin and human serum albumin by spectroscopic methods. J Photochem Photobiol A 179(1-2): 161-166.

16. N Bijari, Y Shokoohinia, M R Ashrafi Kooshk (2013) Spectroscopic study of interaction between osthole and human serum albumin: identification of possible binding site of the compound. Journal of Luminescence 143 328-336.

17. JJ Stephanos (1996) Drug-protein interactions. Two-site binding of heterocyclic ligands to a monomeric hemoglobin. 62(3): 155-169.

18. JJ Stephanos, SA Farina, AW Addison (1996) Iron ligand recognition by monomeric hemoglobins, Biochim. Biophys Acta 1295(2): 209-221.

19. Labban L (2014) The Effects of Rosemary (Rosmarinus officinalis) Leaves Powder on Glucose Level, Lipid Profile and Lipid Perodoxation. International Journal of Clinical Medicine 5(6): 297-304.

20. Ibarra A, Cases J, Roller M, Chiralt Boix A, Coussaert A (2011) Carnosic Acid-Rich Rosemary (Rosmarinus officinalis L.) Leaf Extract Limits Weight Gain Andimproves Cholesterol Levels and Glycaemia in Mice on a High-Fat Diet. British Journal of Nutrition 106(8): 1182-1189.

21. Alnahdi HS (2012) Effect of Rosmarinus officinalis Extract on Some Cardiac Enzymes of Streptozotocin-Induced Diabetic Rats. Journal of Health Sciences 2(4): 33-37.

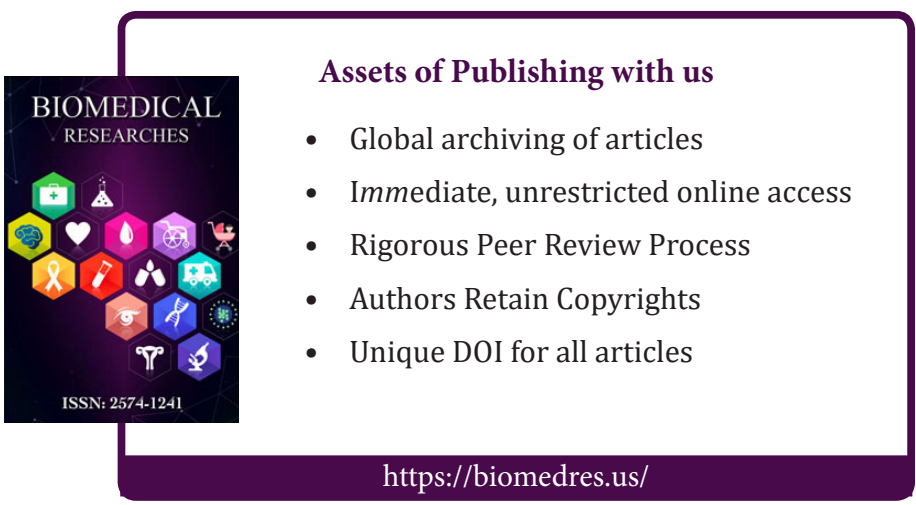

Copyright@ Zeyad J Yassin, Tarek M Zaida | Biomed J Sci \& Tech Res | BJSTR. MS.ID.003921. 